\title{
ChemComm
}

\section{Assembly-driven synthesis of hybrid molecular capsules controlled by chiral sorting $\dagger$}

Cite this: Chem. Commun., 2013
49, 3860

Received 27th February 2013,

Accepted 19th March 2013

DOI: $10.1039 /$ c3cc41515e

www.rsc.org/chemcomm

Chiral capsules with polar interiors (reversed capsules) undergo heterochiral sorting and exhibit positive mutalism - both hemispheres mutually benefit from the association. This feature can be coupled with partial reversibility of the formation reaction and utilized to amplify synthesis of hybrid capsules made of hemispheres that cannot be formed independently.

Non-covalent assembly can contribute considerably to the overall stability of systems, and therefore it can be used as a directing force for numerous chemical processes. Nature often uses molecular recognition to drive the selectivity of its crucial chemical reactions. Chemists have also shown that molecular recognition can be employed to direct synthesis of complex molecular architectures that are not achievable by simple chemoselective synthesis (rotaxanes, knots, etc.,). ${ }^{1,2}$ Dynamical combinatorial chemistry couples stability gained from recognition with reversibility of the chemical reaction. In this way the most stable assemblies have a chance to be amplified. ${ }^{3-6}$ In the current communication we show that the stability gained from non-covalent assembly of capsular dimers can be used to control their own chemical synthesis. Additionally, we show that chiral sorting plays a crucial role in the selectivity of this process. We and others have previously demonstrated that sorting ${ }^{7-12}$ and chiral sorting, in particular, ${ }^{13-17}$ can effectively control assembly of capsules. However, it has not been previously shown that that chiral sorting can also mutually influence the chemical synthesis of hemispheres.

Recently we reported the synthesis of L-2a, which is composed of a rigid resorcin[4] arene scaffold and flexible L-phenylalanine arms (Scheme 1). ${ }^{17}$ The product dimerizes quantitatively in the reaction mixture and it is isolated as capsules (L-2a) $)_{2}$ in a high overall yield (70\%). The self-complementary dimers (L-2a) $)_{2}$ have two unique features: they are chiral and have polar interiors, which makes them very efficient in chiral recognition of small

Institute of Organic Chemistry, Polish Academy of Sciences, Kasprzaka 44/52, 01-224 Warsaw, Poland. E-mail: agnieszka.szumna@icho.edu.pl;

Fax: +4822 6326681; Tel: +48223432101

$\dagger$ Electronic supplementary information (ESI) available: Synthetic and isolation procedures. NMR spectra of all new capsules synthesized. CCDC 918981. For ESI and crystallographic data in CIF or other electronic format see DOI: 10.1039/c3cc41515e

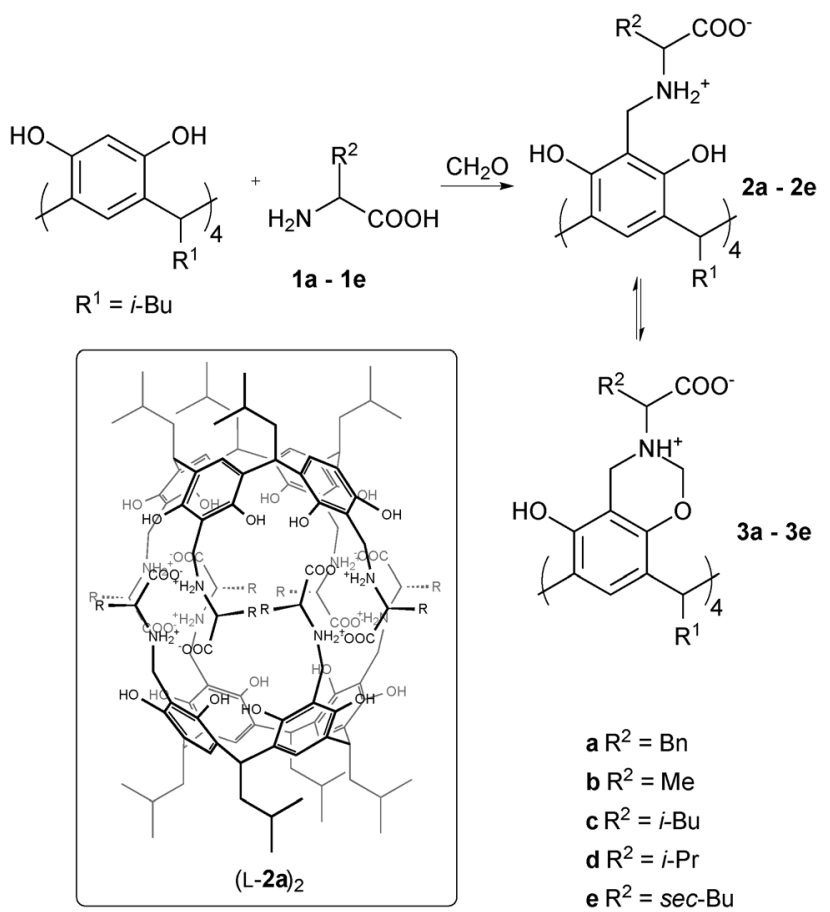

Scheme 1 Synthesis and structure of "reversed" capsules.

polar molecules. ${ }^{18,19}$ Since the binding motif of the dimer (interactions by two belts of salt bridges) involves only amino acid backbones, it can be expected that similar capsules should be formed with other amino acids having hydrophobic side chains. However, we have currently found that using even chemically similar amino acids leads to dramatic changes: not only in the association and complexation properties but also in the outcome of the chemical synthesis. We have found that the assembly process and successful formation of the product by the Mannich reaction are inextricably connected.

We have tested the Mannich reaction with various hydrophobic amino acids: Ala 1b, Leu 1c, Val 1d and Ile 1e. In the case of $\mathbf{L - 1} \mathbf{1 b}$, the dimeric homochiral capsule (L-2b) $)_{2}$ was formed in the reaction medium and isolated in 50\% yield (ESI $\dagger$ ). However, for 
L-1c, an amino acid with a longer and more flexible alkyl chain, the obtained product had a very complicated NMR spectrum with pronounced upfield shifted signals of methyl groups (ESI $\dagger$ ). Further experiments showed that the product existed in a dimeric form (L-2c) $)_{2}$ but one of the leucine side chains was selfencapsulated, thus preventing guest complexation. This was in contrast to (L-2a) $)_{2}$, which in some solvents could also exist in selfencapsulated form but remained fully effective in guest binding. The most surprising behaviour was observed for L-1d. In this case we did not obtain a capsule (L-2d) $)_{2}$. Moreover, despite numerous attempts, the secondary amine L-2d could not be obtained even in its monomeric form, using this reaction.

In order to gain insight into possible reasons for such differences we examined the course of the Mannich reaction between resorcin[4]arene, formaldehyde and various amino acids by NMR (Scheme 1 and Fig. 1). We chose the two borderline cases: L-1a and L-1d. In both cases the reaction initially led to the formation of variously substituted benzoxazines 3 (Fig. 1a and e). The existence of benzoxazine intermediates was confirmed using HSQC and DOSY spectra (ESI + ). For L-1a the signals of benzoxazine species gradually disappeared during the progress of the reaction and finally, after 5 days, the main product was L-2a, which assembled into capsules (L-2a) ${ }_{2}$ (Fig. 1 b and c). However, for L-1d the reaction did not equilibrate to yield the final four-substituted secondary amine product L-2d. Instead, very broad peaks were obtained, indicative of non-specific aggregation (Fig. 1d). After addition of methanol to disassemble the aggregates, the spectrum revealed a complicated mixture of randomly substituted benzoxazines 3 and secondary amines 2 with unreacted aromatic

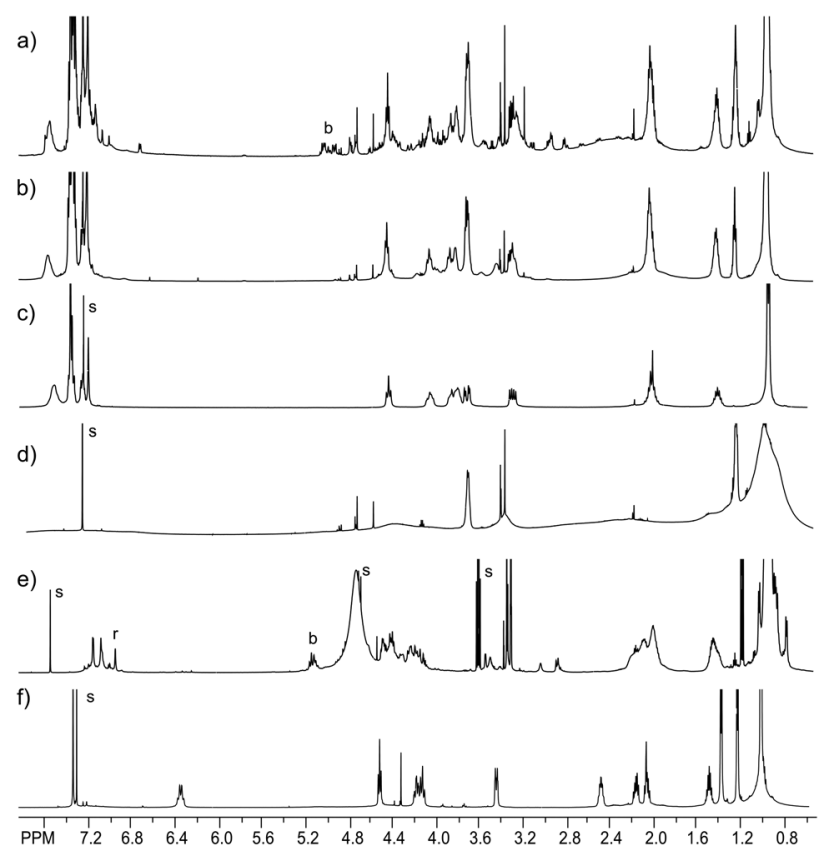

Fig. $1{ }^{1} \mathrm{H}$ NMR spectra (600 MHz) of reaction mixtures: (a) after 1 day from substrate L-1a $\left(\mathrm{CDCl}_{3}\right)$; (b) after 5 days from substrate L-1a $\left(\mathrm{CDCl}_{3}\right)$; (c) final isolated product from substrate $\mathrm{L}-\mathbf{1} \mathbf{a}\left(\mathrm{CDCl}_{3}\right)$; (d) after 5 days from substrate $\mathrm{L}-\mathbf{1} \mathbf{d}$ $\left(C D C l_{3}\right)$; (e) after 5 days from substrate $L-1 d\left(C D C l_{3}: C D_{3} O D 1: 1\right)$; $(f)$ isolated product $(\mathrm{L}-\mathbf{2} \mathbf{d})(\mathrm{D}-\mathbf{2} \mathbf{d})\left(\mathrm{CDCl}_{3}\right) ; \mathrm{s}$ - solvent, $\mathrm{b}$ - benzoxazine, $\mathrm{r}$ - unreacted aromatic positions from resorcinarene.
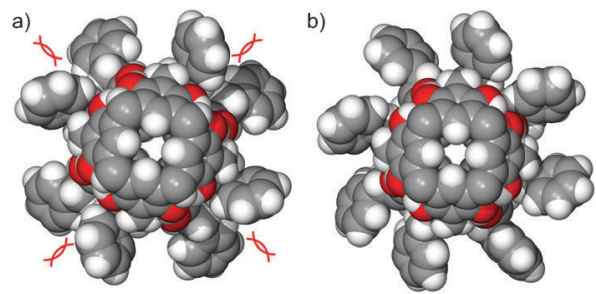

Fig. 2 Top view of X-ray structures of: (a) homochiral dimer (L-2a) $)_{2}$ (Refcode HOWHAD); (b) heterochiral dimer (L-2a)(D-2a). \& denotes steric crowding between phenylalanine side chains (highly disordered $\mathrm{CH}_{2} \mathrm{Cl}_{2}$ and $\mathrm{CH}_{3} \mathrm{NO}_{2}$ molecules were removed by SQUEEZE).

positions (Fig. 1e). Attempts to force the reaction by heating, prolonged reaction times, addition of acidic catalyst $\mathrm{AcOH}$, $10 \%$ ) or addition of methanol (5-50\%) to disassemble aggregates also failed. These results indicate that the formation of benzoxazines 3 is reversible (as also proved by others) ${ }^{20}$ and assembly of a dimeric capsule $(\mathrm{L}-\mathbf{2 a})_{2}$ moves the equilibrium towards secondary amines. Apparently, for L-1d this driving force is missing, and therefore the reversible step of the Mannich reaction does not lead to secondary amine L-2d.

The possible reason why a capsular dimer fails to form can be deduced from the X-ray structure. In homochiral dimer $(\mathrm{L}-2 \mathrm{a})_{2}$ the amino acid side chains are grouped pairwise (Scheme 1 and Fig. 2). ${ }^{17}$ This can create potential sterical conflicts between amino acid side chains, especially branched at the position next to the stereogenic center (like L-Val). However, the steric hindrance can be considerably diminished in a dimer composed of hemispheres of opposite chirality. The $\mathrm{X}$-ray structure of (L-2a)(D-2a) shows that in a heterochiral capsule the amino acid side chains form a pinwheel type of structure that is devoid of steric clashes (Fig. 2b). $\neq$ This results in the formation of a more stable dimer. Indeed, we have previously proven that, in solution, a heterochiral capsule is thermodynamically more stable than a homochiral one. ${ }^{17}$

Based on this structural information we have predicted that, even though we cannot obtain a homochiral capsule $(\mathrm{L}-2 \mathbf{d})_{2}$ for steric reasons, the formation of a heterochiral capsule (L-2d)(D-2d) should be possible and can therefore possibly drive the reversible step of the Mannich reaction. This observation prompted us to develop a strategy for obtaining hybrid capsules ${ }^{21-24}$ that are not accessible through a traditional sequence: chemical reaction $\rightarrow$ isolation of homochiral capsules $\rightarrow$ association of heterochiral capsules. We took advantage of the reversibility of benzoxazine formation and the higher thermodynamic stability of heterochiral arrangement. Therefore, we tested the effectiveness of assemblydriven synthesis at the different levels (Fig. 3) and under various reaction conditions. $\S$ First we used a pre-formed homochiral capsule $(\mathrm{L}-2 \mathrm{a})_{2}$ as a template for formation of a heterochiral hybrid capsule with a D-1d partner (Fig. 3a). The product (L-2a)(D-2d) was isolated in 45\% yield. This result finally proves that the assembly can drive the synthesis and also eliminates the possibility that inherent reactivity of valine is responsible for the negative outcome of the Mannich reaction. We then investigated whether we could obtain a hybrid capsule without a pre-formed template. In the first approach the reactions were carried out so 


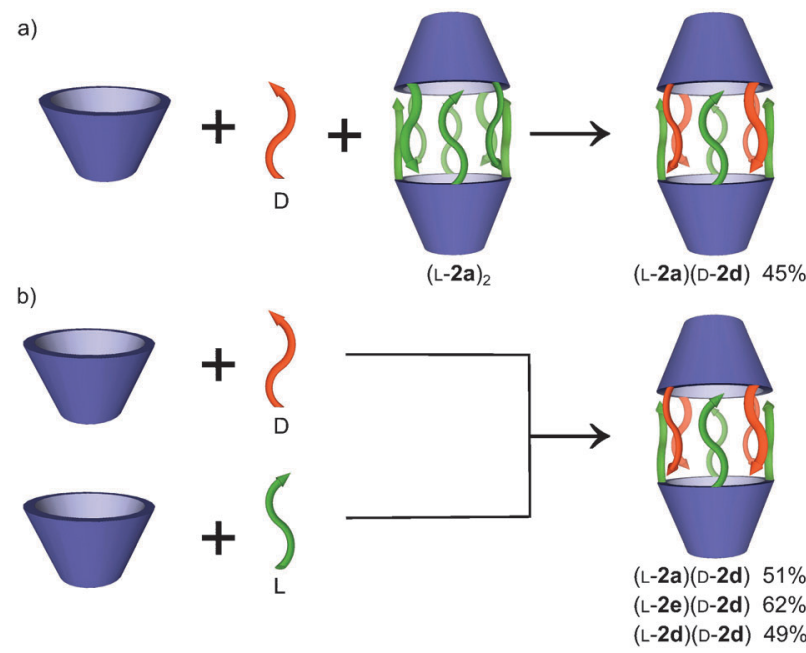

Fig. 3 Sorting experiments in the synthesis of hybrid capsules.

that the first step took place separately for opposite enantiomers. Then the reaction mixtures were combined and the mixture was allowed to assemble and equilibrate (Fig. 3b). In this way the test capsule (L-2a)(D-2d) was obtained in 51\% yield. The strategy has proven to be effective for the synthesis of new capsules made of two monomers, of which none was obtained as a single component, for example (L-2e)(D-2d) (62\%) and (L-2d)(D-2d) (49\%). Finally, a totally one pot procedure using racemic amino acids was tested. However, the limitation of this approach is the first reaction step (attachment to the resorcinarene skeleton) which, under mild conditions (required for effective assembly), has limited (or no) reversibility. Therefore, the one-pot procedure proved to be ineffective.

As the molecular capsules with reversed polarity are already formed in the reaction medium, their isolation also requires special care to protect their secondary structure. Similarly to natural biological polymers, if the capsular dimers are not properly handled, they are prone to denaturation, which destroys their function. Dimers (L-2b) $)_{2}$ are stable and easily isolated in non-polar solvents. However, when $(\mathrm{L}-\mathbf{2 b})_{2}$ was dissolved in DCM-MeOH and evaporated, its capsular properties were lost. As a result of denaturation the material was no longer soluble in non-polar solvents and did not show complexing properties. This denaturation process was to some extent reversible, but it required days (in chloroform) for (L-2b $)_{2}$ to partially restore its capsular structure. However, some part of the material remained irreversibly denaturated. The plausible explanation involves formation of non-covalent oligomeric polymers instead of well ordered dimers as the polarity of solvent increases (DCM evaporates faster than methanol). Although the dimers are thermodynamically favoured in non-polar solvents, their formation is slow and therefore re-denaturation is very slow. The denaturation process is most pronounced for capsules that have "thin" hydrophobic shells e.g. (L-2b) $)_{2}$. For "thicker" hydrophobic shells, e.g. $(\mathrm{L}-2 \mathrm{a})_{2}$, denaturation still occurs, but to a lesser extent. This observation has important implications from the synthetic point of view. Improper reaction conditions or improper work-up destroys supramolecular assemblies and may result in a chemical compound, but not a functional material. Since the self-assembly process is very slow, the real value of the newly synthesized compound can be easily overlooked.

In conclusion, we have shown that the chemical synthesis of capsules with reversed polarity is driven by their self-assembly. The hemispheres can mutually promote their own formation. Therefore, it is possible to obtain a capsule made of two different hemispheres even if none of them can be synthesized separately. Both formation by chemical reaction and successful isolation are inextricably connected with formation of dimers. If the capsules are not formed either due to steric requirements or due to denaturation, the products cannot be obtained, even as monomers. Higher thermodynamic stability of heterochiral dimers can be employed to drive the chemical reaction of their formation. The new hybrid dimers obtained by this approach exhibit positive mutalism - they stabilise their own formation and cannot exist separately.

We acknowledge the financial support from the National Science Centre (Grant No. N204 187839).

\section{Notes and references}

‡ Crystallographic data for $(\mathrm{L}-2 \mathrm{a})(\mathrm{D}-2 \mathrm{a}) \times 6 \mathrm{CH}_{2} \mathrm{Cl}_{2} \times 6 \mathrm{CH}_{3} \mathrm{NO}_{2}$ : $\mathrm{C}_{180} \mathrm{H}_{230} \mathrm{Cl}_{12} \mathrm{~N}_{14} \mathrm{O}_{44} M=3719.18,0.63 \times 0.76 \times 0.93 \mathrm{~mm}^{3}$, monoclinic, space group $P 2_{1} / n$ (no. 14), $a=17.9860(7), b=32.4038(14), c=$ 32.9781(14) $\mathrm{A}, \beta=90.406(2)^{\circ}, V=19219.6(14) \AA^{3}, Z=4, D_{\mathrm{c}}=1.285 \mathrm{~g} \mathrm{~cm}^{-3}$, $F_{000}=7856$, CuK $\alpha$ radiation, $\lambda=1.54184 \AA, T=173(2) \mathrm{K}, 2 \theta_{\max }=136.1^{\circ}$, 415267 reflections collected, 34285 unique $\left(R_{\text {int }}=0.0690\right)$. Final GooF $=$ $1.035, R_{1}=0.0700, \mathrm{w} R_{2}=0.1917, R$ indices based on 28496 reflections with $I>2 \sigma(I)$ (refinement on $F^{2}$ ), 1860 parameters, 0 restraints. Lp and absorption corrections applied, $\mu=2.227 \mathrm{~mm}^{-1}$. Squeeze procedure has been applied to remove highly disordered solvent molecules. CCDC 918981.

$\S$ See ESI $\dagger$ for details.

1 A. Bogdan, M. O. Vysotsky, T. Ikai, Y. Okamoto and V. Böhmer, Chem.-Eur. J., 2004, 10, 3324.

2 A. Bogdan, Y. Rudzevich, M. O. Vysotsky and V. Böhmer, Chem. Commun., 2006, 2941.

3 Z. Rodriguez-Docampo, E. Eugenieva-Ilieva, C. Reyheller, A. M. Belenguer, S. Kubik and S. Otto, Chem. Commun., 2011, 47, 9798.

4 L. Vial, R. F. Ludlow, J. Leclaire and S. Otto, J. Am. Chem. Soc., 2006, 128, 10253.

5 S. Otto, Acc. Chem. Res., 2012, 45, 2200.

6 K. Oh, K. S. Jeong and J. S. Moore, Nature, 2001, 414, 889.

7 O. Mogck, V. Böhmer and W. Vogt, Tetrahedron, 1996, 52, 8489.

8 H. Gan and B. C. Gibb, Chem. Commun., 2012, 48, 1656.

9 A. S. Singh and S. S. Sun, Chem. Commun., 2012, 48, 7392.

10 M. M. J. Smulders, A. Jimenez and J. R. Nitschke, Angew. Chem., Int. Ed., 2012, 51, 6681.

11 E. S. Barrett, T. J. Dale and J. Rebek, Jr, J. Am. Chem. Soc., 2008, 130, 2344.

12 Y. Rudzevich, V. Rudzevich, F. Klautzsch, C. A. Schalley and V. Böhmer, Angew. Chem., Int. Ed., 2009, 48, 3867.

13 M. Chas, G. Gil-Ramirez, E. C. Escudero-Adan, J. Benet-Buchholz and P. Ballester, Org. Lett., 2010, 12, 1740.

$14 \mathrm{~A}$. Wu and L. Isaacs, J. Am. Chem. Soc., 2003, 125, 4831.

15 C. G. Claessens and T. Torres, J. Am. Chem. Soc., 2002, 124, 14522.

16 M. Alajarin, R. A. Orenes, J. W. Steed and A. Pastor, Chem. Commun., 2010, 46, 1394.

17 B. Kuberski and A. Szumna, Chem. Commun., 2009, 1959.

18 A. Szumna, Chem. Commun., 2009, 4191.

19 A. Szumna, Chem.-Eur. J., 2009, 15, 12381.

20 C. Schmidt, E. F. Paulus, V. Böhmer and W. Vogt, New J. Chem., 2000, 24, 123.

21 D. Ajami, M. P. Schramm, A. Volonterio and J. Rebek, Jr., Angew. Chem., Int. Ed., 2007, 46, 242.

22 C. Valdés, U. P. Spitz, L. M. Toledo, S. W. Kubik and J. Rebek, Jr., J. Am. Chem. Soc., 1995, 117, 12733.

23 B. M. O'Leary, T. Szabo, N. Svenstrup, C. A. Schalley, A. Lützen, M. Schäfer and J. Rebek, Jr., J. Am. Chem. Soc., 2001, 123, 11519.

24 R. K. Castellano, B. H. Kim and J. Rebek, Jr., J. Am. Chem. Soc., 1997, 119, 12671. 